\title{
Searches for Anomalous lonizing Particles, SIMPs, and Monopoles at the LHC
}

\author{
Claudia-Elisabeth Wulz, on behalf of the ATLAS and CMS Collaborations ${ }^{a, *}$ \\ ${ }^{a}$ Institute of High Energy Physics, Austrian Academy of Sciences \\ Nikolsdorfergasse 18, 1050 Vienna, Austria \\ E-mail: claudia.wulz@cern.ch
}

Searches for anomalous ionizing particles, strongly interacting massive particles (SIMPs, $\chi$ ), and magnetic monopoles by the ATLAS and CMS Collaborations with proton-proton collisions recorded at the Large Hadron Collider at a centre-of-mass energy of $\sqrt{s}=13 \mathrm{TeV}$ are presented. No evidence for such particles has been found, but lepton-like multi-charged particles with masses between $50 \mathrm{GeV}$ and 980 to $1220 \mathrm{GeV}$ are excluded for electric charge numbers in the range $2 \leq z \leq 7$. SIMP masses less than $100 \mathrm{GeV}$ are excluded for a scalar mediator with couplings to quarks and SIMPs $g_{q}=1, g_{\chi}=-1$. An improvement by a factor five of the constraints on the direct production of magnetic monopoles with one or two magnetic Dirac charges and stable objects with $20 \leq z \leq 60$ was achieved with respect to a previous analysis, and the charge range has been extended to $60 \leq z \leq 100$.

The Ninth Annual Conference on Large Hadron Collider Physics - LHCP2021

7-12 June 2021

Online

\footnotetext{
${ }^{*}$ Speaker
} 


\section{Introduction}

The standard model of particle physics (SM) has undoubtedly been incredibly successful. Yet, we know that it will have to be embedded in a more overarching theory, in order to describe all phenomena unexplained so far, such as dark matter or dark energy. Intriguing possible deviations from its predictions, for example regarding lepton-flavour violation, might pave the way. One can also look for exotic particles not present in the SM, such as anomalous ionizing particles, strongly interacting massive particles (SIMPs), or magnetic monopoles. Recent searches for such particles have been undertaken both at the ATLAS [1] and CMS [2] experiments, and are described in the following.

\section{Heavy long-lived multi-charged particles}

Several models predict heavy long-lived multi-charged particles. Examples are AC-leptons pairs of SU(2) electroweak singlets with opposite charges and no other gauge charges of the SM, which makes them behave as heavy stable charged leptons - predicted by the almost-commutative model [3], or technibaryons, made of two techniquarks or two anti-techniquarks with an arbitrary value of the electric charge, predicted by the walking-technicolor model [4]. Other signatures could come from doubly charged Higgs bosons in a left-right symmetric model [5], or other doubly charged particles [6] that could explain the positron excess in dark matter searches. The signature we are looking for is governed by ionization, described by the Bethe-Bloch formula, which states that the energy loss per unit length $d E / d x$ is proportional to $z^{2}$, where the absolute electric charge is given by $|q|=z e$.

A search for heavy long-lived multi-charged particles has been performed by the ATLAS Collaboration [7], using $36.1 \mathrm{fb}^{-1}$ of LHC proton-proton data. The analysis considered particles with charges in half-integer steps, in the charge range $2 \leq z \leq 7$. The production model assumes purely electromagnetic couplings, proportional to the charge of the multi-charged particles, in the mass range between 50 to $1400 \mathrm{GeV}$. These muon-like particles traverse the ATLAS detector without decaying in its volume. Their large energy loss leads to their slow-down, which is why trigger and reconstruction need to be adapted. A single-muon trigger with relative transverse momentum $p_{T} / z>50 \mathrm{GeV}$ was used, which is sensitive for particles travelling at velocities $\beta>0.6$. In order to increase the data sample by about $20 \%$, a missing transverse calorimeter energy trigger of $E_{\mathrm{T}}^{\text {miss }}>70,90$, or $110 \mathrm{GeV}$, depending on the data taking period, has been added. In order to select candidate events, the ionization energies measured in the pixel detector, the transition radiation tracker (TRT), and the monitored drift tube (MDT) chambers have been considered. Ionization estimators based on a tight and the final selection have been defined. The significance $\mathrm{S}(d E / d x)$ was estimated by comparison with the average for a highly-relativistic muon using a $Z \rightarrow \mu \mu$ control sample, as can bee seen in Fig. 1 (left). Background sources taken into account were muons with ionization randomly fluctuating to larger values due to occupancy effects, and $\delta$-rays. Two methods of background estimation have been applied. In the first one, for $z=2$, an ABCD method was used, with the plane $\mathrm{S}$ (TRT $d E / d x$ ) - S (MDT $d E / d x$ ) divided in four regions, using the final selection cuts, as shown in Fig. 1 (right). The expected number of background events in the signal region $\mathrm{D}$ was estimated from observed data events in the regions $\mathrm{A}, \mathrm{B}, \mathrm{C}$. For $z \geq 2$ a sideband 
method was used. The considered uncertainties consist of the background estimation uncertainty ( $67 \%$ for $z=2,75 \%$ for $z>2$ ), the uncertainty on the integrated luminosity (2.1\%), and the signal selection efficiency as a function of $z$ and particle mass, convoluted from the uncertainty on the data-simulation comparison, the trigger efficiency, and the PDF parameterization.
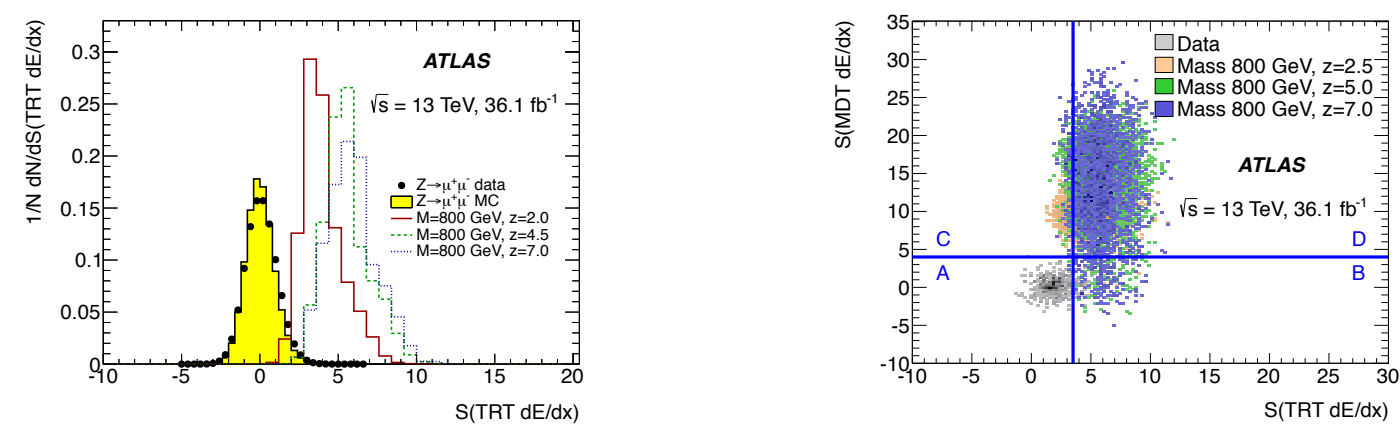

Figure 1: Normalized distributions of the $d E / d x$ significance in the TRT, S(TRT $d E / d x$ ) for muons from $\mathrm{Z} \rightarrow \mu \mu$ (data and simulation) and for simulated multi-charged particles passing preselection requirements (left). S(MDT $d E / d x$ ) versus S(TRT $d E / d x$ ) after the $z>2$ selections (right).

Lepton-like multi-charged particles with masses between $50 \mathrm{GeV}$ and 980 to $1220 \mathrm{GeV}$ are excluded for $2 \leq z \leq 7$, as can be seen from Fig. 2 .
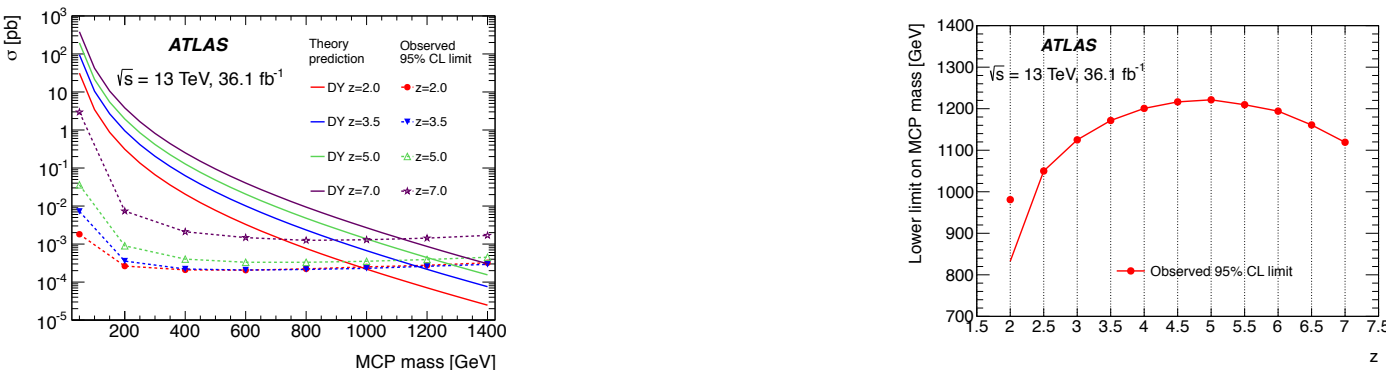

Figure 2: Observed 95\% CL cross section upper limits and theoretical cross sections for a Drell-Yan pairproduction model as functions of the lepton-like multi-charged particle's mass for several values of $z$ between 2 and 7 (left). Observed 95\% CL lower mass limits of lepton-like multi-charged particles (right).

\section{Strongly interacting massive particles}

Searches for weakly interacting massive particles (WIMPs) have not been successful so far, therefore strongly interacting massive particles (SIMPs, $\chi$ ), with their large interaction cross sections with nucleons are now increasingly targeted. A CMS analysis [8] with $16 \mathrm{fb}^{-1}$ of $\sqrt{s}=13 \mathrm{TeV}$ data provided the first results for such particles at a collider. It assumes a simplified model, where the interaction occurs through a scalar or vector low-mass mediator. To avoid bound states, a purely repulsive SIMP-nucleon interaction is assumed. SIMPs hadronize only through production of quarks via a mediator radiated by a SIMP. The experimental signature consists of pairs of trackless jets recorded in the calorimeters, with no charged particle tracks in the tracker. A single-jet online 
trigger was used. Offline two jets with $p_{\mathrm{T}}>550 \mathrm{GeV}$ in the tracker volume, separated in azimuth, and with a small fraction of charged particles $(\mathrm{ChF}<0.05)$ were selected. The dominant QCD multijet background was estimated from data. The SIMP interaction in the detector was assumed to be neutron-like. Fig. 3 (left) shows the distributions of the charged fraction for different SIMP masses and simulated QCD events. From Fig. 3 (right) it can be seen that SIMP masses less than $100 \mathrm{GeV}$ are excluded. An upper limit on the fiducial cross section of $0.18 \mathrm{fb}$ at $95 \% \mathrm{CL}$ is also provided for a generic signal of high-momentum trackless jets. For masses above $100 \mathrm{GeV}$, where the modelling of the SIMP-nucleon interaction becomes more speculative, the obtained cross section upper limits are increasingly uncertain.
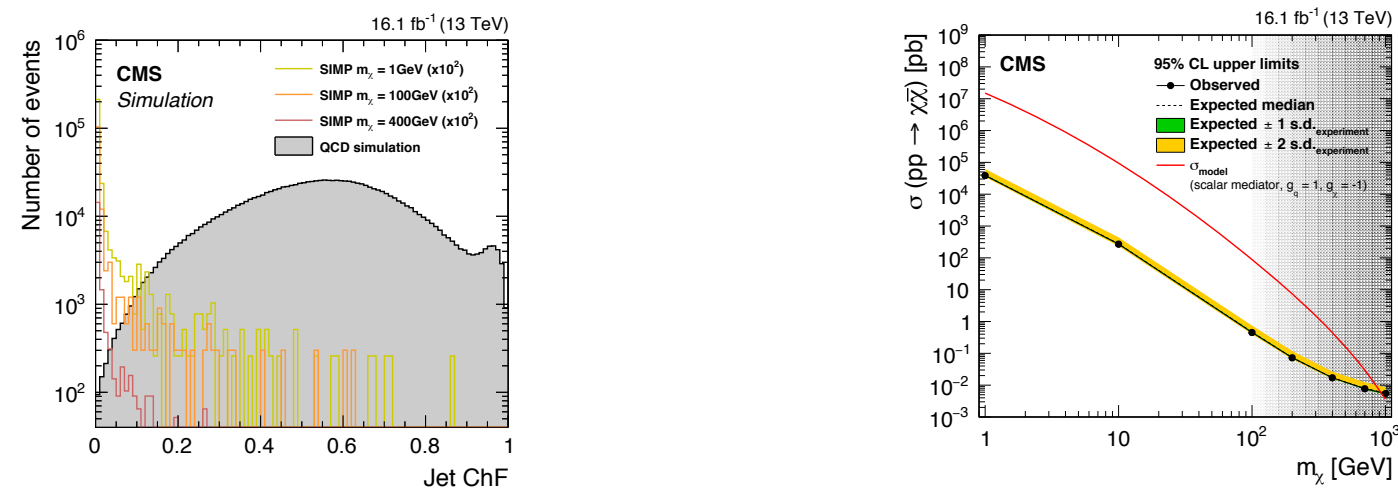

Figure 3: Value of the charged fraction of the two leading jets (left). Expected and observed 95\% CL upper limits on the production cross section for SIMPs with masses between 1 and $1000 \mathrm{GeV}$ (right). The shaded area indicates the region where the modelling becomes more speculative.

\section{Magnetic monopoles and highly-charged objects}

A high-velocity Dirac monopole of magnetic charge $|\mathrm{g}|=\mathrm{g}_{\mathrm{D}}$, the Dirac charge, would interact with matter like an ion of electric charge $|z|=68.5$. Since the energy loss is proportional to the square of the charge, a monopole with $|\mathrm{g}|=\mathrm{g}_{\mathrm{D}}$ would deposit 4700 times more energy by ionization than a proton, and $\delta$-rays would emerge due to the high stopping power. The high-ionization signature would also be present for stable high-electric-charge (HECO) objects, such as aggregates of ud- or s-quark matter, Q-balls, or micro-black-hole remnants. Electroweak monopoles with masses in the $\mathrm{TeV}$ range are predicted by some models.

ATLAS performed a search for magnetic monopoles and HECOs, collectively referred to as highly ionizing particles, or HIPs, using $34.4 \mathrm{fb}^{-1}$ of $13 \mathrm{TeV}$ proton-proton data [9], with sensitivity up to $|\mathrm{g}|=2 \mathrm{~g}_{\mathrm{D}}$, complementary to MOEDAL, the Monopole and Exotics Detector at the LHC. Results were interpreted for models with spin-0 and spin-1/2 Drell-Yan production of stable particles with one or two Dirac magnetic charges or an electric charge within $20 \leq z \leq 100$ and masses between 200 and $4000 \mathrm{GeV}$. The signature consists of a single HIP in the TRT and a pencil-shaped deposit in the electromagnetic calorimeter (ECAL). Backgrounds from overlapping charged particles in the TRT, high-energy electrons in the ECAL, and noise in TRT straws and the ECAL were estimated from data. Some results on monopoles and HECOs are shown in Fig. 4. An 
improvement by a factor five of the constraints on the direct production of magnetic monopoles with one or two magnetic Dirac charges and stable objects with electric charge numbers in the range $20 \leq z \leq 60$ was achieved with respect to a previous ATLAS analysis [10], and the charge range has been extended to $60 \leq z \leq 100$.
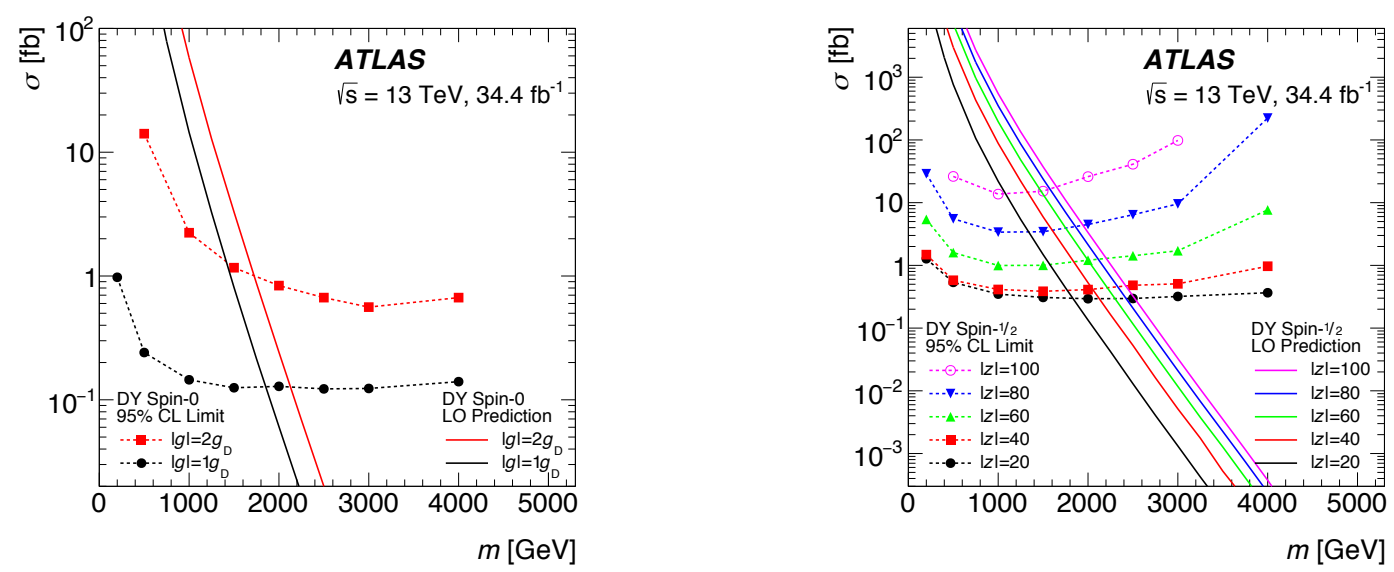

Figure 4: Observed 95\% CL upper limits and predictions for Drell-Yan spin-0 monopole production cross sections (left). Observed 95\% CL upper limits and predictions for Drell-Yan spin-1/2 HECO production cross sections.

\section{References}

[1] G. Aad et al. [ATLAS], JINST 3 (2008), S08003

[2] S. Chatrchyan et al. [CMS], JINST 3 (2008), S08004

[3] C. A. Stephan, J. Phys. A 40 (2007), 9941 [arXiv:0706.0595 [hep-th]]

[4] F. Sannino and K. Tuominen, Phys. Rev. D 71 (2005), 051901 [arXiv:hep-ph/0405209 [hep$\mathrm{ph}]]$

[5] R. N. Mohapatra and J. C. Pati, Phys. Rev. D 11 (1975), 566-571 doi:10.1103/PhysRevD.11.566 Phys. Rev. D 11 (1975), 566-571

[6] C. S. Aulakh, K. Benakli and G. Senjanovic, Phys. Rev. Lett. 79 (1997), 2188-2191 [arXiv:hepph/9703434 [hep-ph]]

[7] M. Aaboud et al. [ATLAS], Phys. Rev. D 99 (2019) no.5, 052003 [arXiv:1812.03673 [hep-ex]]

[8] A. Tumasyan et al. [CMS], [arXiv:2105.09178 [hep-ex]]

[9] G. Aad et al. [ATLAS], Phys. Rev. Lett. 124 (2020) no.3, 031802 [arXiv:1905.10130 [hep-ex]]

[10] G. Aad et al. [ATLAS], Phys. Rev. D 93 (2016) no.5, 052009 [arXiv:1509.08059 [hep-ex]] 